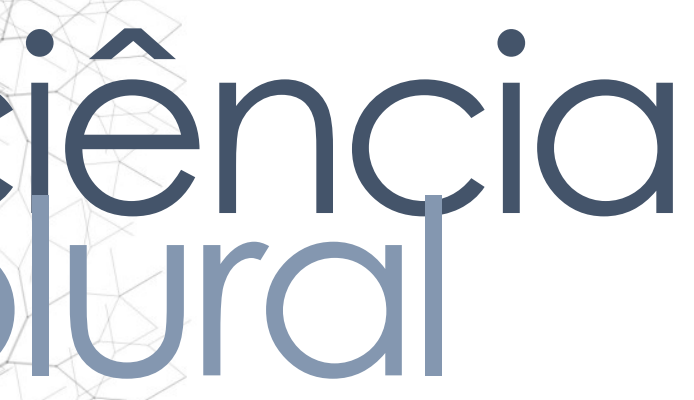

\title{
EVOLUÇÃO DE GRAU DE INCAPACIDADE FÍSICA EM PESSOAS COM HANSENÍASE: ESTUDO LONGITUDINAL
}

Evolution of degree of physical disability in people with leprosy: longitudinal study

Evolución del grado de discapacidad física en personas con leprosía: estudio longitudinal

Giovanna de Oliveira Libório Dourado • Doutora em Enfermagem Professora da Universidade Federal do Piauí • E-mail: giovannaliborio@ufpi.edu.br

Kelly Saraiva dos Santos • Discente do curso de Enfermagem da Universidade Federal do Piauí •E-mail: kellysaraiva2013@gmail.com

Lidya Tolstenko Nogueira • Doutora em Enfermagem Professora da Universidade Federal do Piauí • E-mail: lidyatn@gmail.com

Autora responsável pela correspondência:

Giovanna de Oliveira Libório Dourado • E-mail: giovannaliborio@ufpi.edu.br 


\section{RESUMO}

Introdução: A hanseníase é um agravo infectocontagioso e incapacitante em que mesmo após a alta por cura pode suceder o desenvolvimento de sequelas. Objetivo: avaliar a evolução do grau de incapacidade física (GIF) de pessoas com hanseníase. Método: Trata-se de uma pesquisa observacional, descritiva, de delineamento longitudinal. $\mathrm{O}$ estudo foi desenvolvido em duas etapas com interstício mínimo de dois anos entre as etapas em um município hiperendêmico do Piauí. Foram avaliadas 256 pessoas e analisou-se a forma clínica, classificação operacional e o grau de incapacidade física. Resultados: Os participantes do estudo apresentavam forma clínica indeterminada $(41,8 \%)$, classificação operacional paucibacilar $(55,5 \%)$, no primeiro momento $54 \%$ apresentavam GIF I e no segundo momento 63,5\% também GIF I. A avaliação longitudinal evidenciou aumento entre os classificados como GIF I de 17,64\%. Conclusões: A avaliação dos dois momentos possibilitou constatar que houve aumento entre as pessoas classificadas com algum grau de incapacidade física, ocorrendo aumento percentual entre o GIF I.

Palavras-Chave: Hanseníase, Incapacidade física, Doenças Negligenciadas, Doenças Endêmicas, Saúde Pública.

\section{ABSTRACT}

Introduction: Leprosy is a disabling and contagious infectious disease in which even after discharge by cure can develop the development of sequelae. Objective: To evaluate the evolution of the degree of physical disability of people with leprosy. Method: This is an observational, descriptive, longitudinal design research. The study was developed in two stages with a minimum interstitial of two years between the stages in a hyperendemic municipality of Piauí. 256 people were evaluated and the clinical form, operational classification and the degree of physical disability were analyzed. Results: The study participants had an indeterminate clinical form (41.8\%), paucibacillary operating classification $(55.5 \%)$, at first $54 \%$ had degree of physical disability I and at second moment $63.5 \%$ also degree of physical disability I. Longitudinal evaluation showed an increase among those classified as degree of physical disability I of $17.64 \%$. Conclusions: The evaluation of both moments showed that there was an increase among people classified as having some degree of physical disability, with a percentage increase between degree of physical disability I.

Keywords: Leprosy, physical disability ,Neglected Diseases Endemic Diseases, Public Health.

\section{ESUMEN}

troducción: la lepra es una enfermedad infecciosa e incapacitante en la cual, incluso después del alta debido a la cura, puede ocurrir el desarrollo de secuelas. Objetivo: evaluar la evolución del grado de discapacidad física (GIF) de las personas con lepra. todo: Este es un estudio observacional, descriptivo, longitudinal. El estudio se desarrolló en dos etapas con un intersticio mínimo de dos años entre etapas en un municipio hiperendémico de Piauí. Se evaluaron 256 personas y se analizó la forma 


\section{ciência
purál}

clínica, la clasificación operativa y el grado de discapacidad física. Resultados: los participantes del estudio tenían una forma clínica indeterminada (41.8\%), clasificación operacional paucibacilar (55.5\%), 54\% tenían GIF I en el primer momento y $63.5 \%$ también GIF I. La evaluación longitudinal mostró un aumento entre los clasificados como GIF I del 17,64\%. Conclusiones: La evaluación de los dos momentos permitió verificar que hubo un aumento entre las personas clasificadas como que tenían algún grado de discapacidad física, con un aumento porcentual entre GIF I.

Palabras clave: Lepra, discapacidad física, enfermedades desatendidas, enfermedades endémicas, salud pública. 


\section{Introdução}

A hanseníase é uma doença infectocontagiosa de caráter crônico que acomete principalmente a pele e os nervos periféricos gerando impactos de diferentes naturezas ${ }^{1}$. Possui distribuição universal e faz parte do grupo das doenças tropicais negligenciadas, constituindo-se um grande problema social, visto que as condições sanitárias contribuem para persistência da doença, fato que perpetua os ciclos da pobreza e marginalização ${ }^{2,3}$.

A OMS constatou que a hanseníase permanece sendo um agravo de saúde em diversas partes do mundo. Com relação ao diagnóstico de casos novos, no ano de 2016, destacam-se três regiões: o Sudeste da Ásia, o continente Americano e África. A Índia apresentou a maior quantidade de casos novos, seguida do Brasil e Indonésia; e esses três países representam $82,6 \%$ de todos os casos mundiais registrados no ano de $2016^{4}$.

A prevalência de casos de hanseníase no Brasil em 2015 foi de 1,01/10.000 habitantes, a menor registrada em 11 anos. As regiões Norte, Nordeste e Centro-Oeste apresentaram coeficiente de prevalência acima da média nacional e explicam a carga endêmica do país no ano em questão ${ }^{5}$.

O Piauí, área hiperendêmica, apresentou em 2016 o coeficiente geral de detecção (CGD) de 28,04/100.000 habitantes, considerado muito alto ${ }^{6}$. No Piauí, dentre os diagnosticados com hanseníase, 1,67\% foram classificados como grau de incapacidade física (GIF) II. Em Teresina 2,7\% foram diagnosticados com algum GIF e em Floriano $3,39 \%$ dos casos diagnosticados possuíam alguma incapacidade ${ }^{7}$. A cidade de Floriano, no período de 2001 a 2016, foi classificada como hiperendêmica para hanseníase, no município, em 2016 o CGD foi de 45,75/100.000 habitantes ${ }^{8}$.

O diagnóstico da hanseníase é clínico, epidemiológico e quando necessário laboratorial (baciloscopia e biópsia de pele) ${ }^{9}$. É realizada avaliação dermatoneurológica que investiga alteração de sensibilidade na pele e/ ou lesões; acometimento neural com ou sem espessamento, associado a alterações sensitivas, toras e autonômicas; e baciloscopia positiva e realiza a classificação do GIF ${ }^{10}$.

É importante destacar que o GIF pode sofrer alterações, por isso a sua ssificação não deve ser restrita ao momento do diagnóstico. Dados de coorte ospectiva com pessoas com hanseníase com incapacidades em Belo Horizonte, 
entre 1993 e 2003, destacaram que 43,2\% apresentavam grau 1 na primeira avaliação e, posteriormente, evoluíram para grau 0. Entre aqueles classificados em grau 2 houve melhora, 21,3\% passaram a ter grau 0 e $20 \%$ grau 1 . Para a melhoria na evolução clínica e prevenção de incapacidades físicas, pode-se associar investigação e diagnóstico precoce das neuropatias, intervenções não medicamentosas, técnicas de prevenção de incapacidade e uso de dosagens adequadas de corticoterapia ${ }^{11}$.

As pessoas que têm maior risco de desenvolver deficiências são as que apresentam GIF 1 ou 2 no diagnóstico. O percentual das pessoas que iniciam o tratamento com grau 2 é bastante alto, evidenciando o diagnóstico tardio da hanseníase no Brasil12.

Ao considerar que a hanseníase é uma doença que pode ocasionar sequelas e incapacidades mesmo após a conclusão do tratamento é essencial que após a alta exista longitudinalidade do cuidado, e que a pessoa continue sendo acompanhada pela estratégia saúde da família (ESF). Diante do contexto apresentado, o objetivo do presente estudo é avaliar a evolução do grau de incapacidade física (GIF) de pessoas com hanseníase.

\section{Metodologia}

Trata-se de uma pesquisa observacional, descritiva, de delineamento longitudinal. $\mathrm{O}$ estudo foi desenvolvido em duas etapas com interstício mínimo de dois anos entre as etapas. A primeira ocorreu atrelada a um projeto de pesquisa operacional do Programa de Pós-Graduação em Enfermagem da Universidade Federal do Piauí, intitulado "IntegraHans Piauí: Abordagem integrada de aspectos clínicos, epidemiológicos (espaço-temporais), operacionais e psicossociais da hanseníase em municípios piauienses de alta endemicidade". A segunda etapa insere-se dois anos depois, com nova coleta de dados com as pessoas acometidas por hanseníase que

participaram da primeira etapa e que permaneciam residindo na mesma cidade.

O estudo foi desenvolvido em Floriano, município hiperendêmico do Piauí. A dade de Floriano está distante $244 \mathrm{~km}$ da capital do Estado e se situa à margem eita do Rio Parnaíba, na divisa com o estado do Maranhão. É um importante polo 
comercial, educacional e de saúde da região e entroncamento rodoviário do estado. Tinha uma população de 58.969 habitantes em 2017, predominantemente urbana ${ }^{13}$.

$\mathrm{Na}$ primeira avaliação, buscou-se investigar todos os casos confirmados de hanseníase notificados no Sistema de Informação de Agravos de Notificação (SINAN), no período de 2001 a 2014, residentes no município de Floriano. Foram registrados 1.076 casos, foi critério de inclusão do estudo: possuir idade acima de 18 anos, e não apresentar demência ou condição cognitiva que impossibilitasse responder aos instrumentos. Do total de casos registrados no SINAN, foram localizadas e abordadas 416 pessoas, das quais 256 atendiam aos critérios de inclusão.

Na segunda avaliação, buscou-se todos os incluídos no primeiro momento e foram excluídos aqueles com déficit cognitivo, em viagem para tratamento de câncer, mudança de endereço, óbito, recusa e não localização em domicílio após três tentativas de contato presencial. Assim, das 256 pessoas avaliadas no primeiro momento, foram reavaliadas 189 pessoas, e foi realizado pareamento da amostra.

Analisou-se três variáveis: forma clínica, classificação operacional e grau de incapacidade física. A classificação de Madri estabelece como forma clínica da hanseníase: indeterminada, tuberculoide, dimorfa e virchowiana. A classificação operacional é uma diretriz que orienta a poliquimioterapia (PQT), em que as formas indeterminadas e tuberculoide são formas paucibacilares (PB) da doença, enquanto que a dimorfa e virchowiana são multibacilares (MB) ${ }^{9}$.

Para a avaliação do GIF foram utilizadas as diretrizes atuais, propostas pelo Ministério da Saúde ${ }^{10}$. Assim, os dados coletados na primeira etapa foram revisados e reavaliados conforme a nova classificação, e os dados da segunda etapa foram categorizados considerando as diretrizes em vigor conforme quadro abaixo. 
Quadro 1. Classificação do grau de incapacidade física de acordo com o Ministério da saúde. Brasil (2016).

\begin{tabular}{|c|c|c|}
\hline GIF 0 & GIF I & GIF II \\
\hline $\begin{array}{l}\text { Nenhum } \\
\text { problema com } \\
\text { olhos, mãos e } \\
\text { pés devido à } \\
\text { hanseníase; }\end{array}$ & $\begin{array}{l}\text { Diminuição da força muscular sem } \\
\text { deficiências visíveis das pálpebras ou } \\
\text { perda da sensibilidade da córnea, } \\
\text { resposta demorada ou ausente ao toque } \\
\text { do fio dental ou diminuição/ausência } \\
\text { do piscar. } \\
\text { Mãos: diminuição da força muscular } \\
\text { das mãos sem deficiências visíveis } \\
\text { e/ou alteração da sensibilidade palmar: } \\
\text { não sente o monofilamento } 2 \text { g (lilás) } \\
\text { ou o toque da ponta de caneta } \\
\text { esferográfica. } \\
\text { Pés: diminuição da força muscular dos } \\
\text { pés sem deficiências visíveis e/ou } \\
\text { alteração da sensibilidade plantar: não } \\
\text { sente o monofilamento } 2 \text { g (lilás) ou } \\
\text { o toque da ponta de caneta } \\
\text { esferográfica. }\end{array}$ & $\begin{array}{l}\text { Apresenta lagoftalmo e/ou ectrópio, } \\
\text { triquíase, opacidade da córnea central; } \\
\text { acuidade visual menor que } 0,1 \text { ou } \\
\text { incapacidade de contar dedos a } 6 \\
\text { metros de distância, as mãos } \\
\text { apresentam lesões tróficas e/ou lesões } \\
\text { traumáticas; garras; reabsorção; mão } \\
\text { caída, e os pés, lesões tróficas e/ou } \\
\text { traumáticas; garras; reabsorção; pé } \\
\text { caído e contratura do tornozelo }\end{array}$ \\
\hline
\end{tabular}

Fonte: BRASIL, 2016.

Os dados são apresentados descritivamente em tabelas e para verificar alteração no GIF entre os momentos foi realizado o cálculo de variação estatística. Os aspectos éticos foram respeitados, conforme recomendações da Resolução 466/12 do Conselho Nacional de Saúde.

\section{Resultados}

A tabela 1 apresenta a categorização dos participantes do estudo, em que revaleceu a forma clínica indeterminada (41,8\%), classificação operacional ucibacilar $(55,5 \%)$, no primeiro momento 54\% apresentavam GIF I e no segundo mento 63,5\% também GIF I. Com relação a mudança diante a avaliação 
longitudinal, o grau zero obteve redução (36\%), ocorreu aumento entre os classificados como GIF I de 17,64\% e não houve aumento do quantitativo de pessoas classificadas como GIF II.

Tabela 1. Categorização dos participantes de estudo conforme forma clínica, classificação operacional e grau de incapacidade física. Floriano-PI,2019.

$$
\begin{array}{ccc}
1^{\circ} \text { momento } & 2^{\circ} \text { momento } & \text { Variação estatística } \\
n=189 & n=189 &
\end{array}
$$

$\begin{array}{lllll}\mathrm{N} & \% & \mathrm{~N} & \% & \%\end{array}$

Forma clínica

Indeterminada

7941,8

Tuberculoide

Borderline

Virchowiana

Não definida

\section{Classificação operacional}

Paucibacilar

Multibacilar

GIF

Grau 0

Grau 1

Grau 2

$27 \quad 14,3$

$53 \quad 28,1$

$23 \quad 12,1$

$\begin{array}{ll}7 & 3,7\end{array}$

$105 \quad 55,5$

$84 \quad 44,5$

$\begin{array}{llll}50 & 26,4 & 32 & 16,9\end{array}$

$-36$

$102 \quad 54,0 \quad 120 \quad 63,5$

17,64

$\begin{array}{llll}37 & 19,6 & 37 & 19,6\end{array}$

0

\section{Discussão}

A classificação clínica que prevaleceu no estudo foi a indeterminada, uma das formas iniciais da hanseníase. Porém, destaca-se o elevado percentual de formas clínicas borderline e virchowiana, que podem estar relacionadas a diagnóstico tardio.

Esse resultado está em conformidade com os dados do Sistema de Informação Agravos de Notificação (SINAN), no ano de 2017 no Piauí houve prevalência da rma indeterminada, seguida da dimorfa, tuberculóide e virchowiana ${ }^{14}$. Porém em vergência com a literatura, que frequentemente apresenta a forma dimorfa como de ior prevalência ${ }^{15,16,17}$. 
A respeito da classificação operacional, os resultados evidenciam maior proporção entre os classificados como paucibacilar, destoando de estudos realizados em outros estados brasileiros ${ }^{15,16,18}$.

Ao analisar o quantitativo de pessoas classificadas com a forma clínica indeterminada e tuberculóide, constata-se que o somatório dos percentuais das duas formas não coincide com a classificação operacional PB. Como essa informação foi coletada por meio da notificação registrada no SINAN, pode ter ocorrido falha no diagnóstico ou até mesmo no registro da notificação.

As notificações do SINAN de 2017 no estado do Piauí revelam que entre os notificados como MB, prevaleceu a forma clínica dimorfa, seguida pela virchowiana, porém com registros de forma clínica tuberculóide, indeterminada, não classificada e ignorada. Entre aqueles notificados como PB prevaleceram as formas clinicas indeterminada e tuberculóide, porém houve notificação de casos dimorfa, virchowiana, não classificados e ignorados ${ }^{14}$. Assim, infere-se que pode ter ocorrido tratamento inadequado de pessoas com formas clinicas compatíveis com a classificação operacional MB tratados com esquema terapêutico PB.

A maior parte dos participantes do estudo tem algum GIF, o que é incongruente com a classificação operacional que prevalece (PB) é possível inferir que existe erro diagnóstico e casos que deveriam ter sido tratados como MB evoluíram com piora

A ocorrência de incapacidades também figura em outros estudos nacionais, o que representa um problema de saúde pública pelo fato de serem identificadas incapacidades desde o momento do diagnóstico ${ }^{15,19}$. O alto percentual de GIF também aparece em estudo internacional ${ }^{20}$. É importante destacar que o diagnóstico precoce e a efetividade do tratamento reduzem as consequências ao doente, à família, e à cadeia de transmissão da doença ${ }^{19}$.

Coorte retrospectiva que acompanhou 595 pacientes com incapacidades em Belo Horizonte (MG) encontrou que 43,2\% dos que tinham grau 1 na primeira valiação evoluíram para grau 0 , entre os que apresentavam grau 2, 21,3\% passaram a grau 0 e $20 \%$ passaram a grau 1 . A evolução do GIF está associada a neurite, tempo a ocorrência de neurite, número de nervos acometidos, tipo de tratamento terápico e maior dose de prednisona. Na análise multivariada, o principal fator 
que se associou à evolução do grau de incapacidade foi o grau de incapacidade na admissão ${ }^{11}$.

Avaliação da GIF inicial e final ao tratamento com PQT realizada no período entre 1998-2008, envolvendo pessoas com hanseníase, mostrou que após o tratamento houve redução no GIF, e que o número de complicações em cada caso diminuiu após o tratamento 21 .

Estudo que analisou os casos notificados de hanseníase entre 2009 e 2013, em Floriano, encontrou 388 casos notificados, o perfil epidemiológico era de homens, com idade superior a 15 anos, classificados como forma operacional paucibacilar, forma indeterminada e grau 0 de incapacidade física no momento do diagnóstico ${ }^{22}$.

A longitudinalidade da assistência é um aspecto essencial em que a humanização do cuidado deve ser considerada. A humanização na saúde é um meio para contemplar a assistência integral, a troca por meio da escuta qualificada entre profissionais de saúde e usuários reforçam o vínculo ${ }^{24}$. Considerando o contexto da pessoa com hanseníase, em que mesmo após a alta podem ocorrer sequelas ou agravamento, é primordial envolver os aspectos supracitados para garantir a continuidade do cuidado.

Uma das limitações do estudo diz respeito ao fato de a população ter sido encontrada no banco do SINAN e este apresentar informações incompletas que dificultou a localização de todos os casos notificados. Na tentativa de equacionar esse problema buscou-se outros bancos de dados para obtenção desses endereços. Outra limitação foi percebida é com relação ao espaço- temporal entre os dois momentos de estudo, em virtude de mudanças de endereço que inviabilizaram a inclusão de todos os participantes da primeira etapa. Como estratégia de enfrentamento, buscou-se localizar essas pessoas por meio de informações com vizinhos, ACS e profissionais que atuam na $\mathrm{AB}$ de Floriano e, por último, no CADSUS para confirmar a mudança para outra cidade ou estado. 


\section{Conclusões}

Os participantes do estudo eram pessoas que tiveram diagnóstico de hanseníase, forma clínica indeterminada, classificação operacional paucibacilar, com prevalência de GIF I.

A avaliação dos dois momentos possibilitou constatar que houve aumento entre as pessoas classificadas com algum grau de incapacidade física, ocorrendo aumento percentual entre o GIF I.

Os resultados encontrados relacionados ao GIF podem denotar que existe um distanciamento da ESF após a alta por cura, o que pode ocasionar diagnóstico inoportuno de complicações pós-alta. Pode-se inferir que quanto maior o espaço temporal, maior o afastamento da pessoa, o que a torna mais vulnerável à reações e complicações.

\section{Referências}

1. Ministério da Saúde (BR), Secretaria de Vigilância em Saúde. Guia de Vigilância em Saúde. Brasília (DF);2014.

2. Maciel LR, Ferreira IN. A presença da hanseníase no Brasil: alguns aspectos relevantes nessa trajetória. In: Ferreira IN. Hanseníase: avanços e desafios. Brasília (DF): NESPROM; 2014 1:19-40.

3. Ribeiro MA, Albuquerque IMN, Vasconcelos MIO, Dias LKS, Cavalcante ASP. Geoprocessamento em saúde como tecnologia de análise e monitoramento da hanseníase no município de Sobral-Ceará. Rev Baiana de Saúde Pública. 2017 41: 451-465.

4. World Health Organization (WHS), Weekly epidemiological record Relevé épidémiologique hebdomadaire. ANNÉE 2017; 92(35):501-20

5. Ribeiro MDA, Silva JCA, Oliveira SB. Estudo epidemiológico da hanseníase no Brasil: reflexão sobre as metas de eliminação. Rev Panam Salud Publica [internet]. 2018;e42.

Araujo TME. et.al. Integrahans PI: Boletim de vigilância em saúde do estado do auí: hanseníase 2016. Floriano. Universidade Federal do Piauí. 
7. Ministério da Saúde (BR), Sala de Apoio à Gestão Estratégica (SAGE). Morbidade Hanseníase. Brasília (DF); 2018.

8. Araujo TME. et.al. Integrahans PI: Boletim de vigilância em saúde do estado do Piauí: hanseníase 2016. Floriano: Universidade Federal do Piauí. 2018.

9. Ministério da Saúde (BR), Secretaria de Vigilância em Saúde. Departamento de Vigilância das Doenças Transmissíveis. Guia prático sobre a hanseníase. Brasília (DF); 2017.

10. Ministério da Saúde (BR), Secretaria de Vigilância em Saúde. Departamento de Vigilância das Doenças Transmissíveis. Diretrizes para vigilância, atenção e eliminação da Hanseníase como problema de saúde pública: manual técnico operacional. Brasília (DF); 2016.

11. Gonçalves, SD, Sampaio, RF, Antunes, CMF. Fatores preditivos de incapacidade em pacientes com hanseníase. Rev Saude Publica [internet]. 2009;43: 267-74.

12. Ministério da Saúde (BR), Indicadores epidemiológicos e operacionais de hanseníase no Brasil 2000-2011. Brasília (DF): Ministério da Saúde; 2012.

13.Instituto Brasileiro de Geografia e Estatística (IBGE), Panorama demográfico: Floriano (PI). 2017.

14. Ministério da saúde (BR). SAGE - Sala de Apoio à Gestão Estratégica.

Morbidade - Hanseníase. 2018.

15. Santana EMF, Antas EMV, Brito KKG, Silva MA. Profile of leprosy patients in a secondary health care center Rev. enferm. UFPE. 2017;11(11):4404-4409.

16. Campos MRM, Batista AVA, Guerreiro JV. Perfil clínicoepidemiológico dos pacientes diagnosticados com hanseníase na Paraíba e no Brasil, 2008 - 2012. Rev. Bras. Cien. Saúde [internet]. 2018;22(1):79-86.

17. Amoroso SLO, Conto MA, Lima AAM. Perfil clínico e demográfico da hanseníase no município de Cacoal/RO no período entre 2007 a 2016. Revista Eletrônica FACIMEDIT [internet]. $2017 \mathrm{dez}$ 6:79-83.

18. Reis BM, Castro SS, Fernandes LFRM. Limitation of activity and restriction of social participation in relation to age range, gender, and education in people with leprosy. An. Bras. Dermatol. 2017;92:335-339

Souza EA, Boigny RN, Ferreira AF, Alencar CH, Oliveira MLW, Ramos Jr. NA. lnerabilidade programática no controle da hanseníase: padrões na perspectiva de hero no Estado da Bahia, Brasil. Cad. Saúde Pública. 2018;34(1). 
20. Domple VK, Manoj YH, Satish KW, Rambhau DG, Vishal SD. Assessment of Disability amongst Leprosy Patients: A CrossSectional Study. National Journal of Community Medicine. 2017;8:482-486.

21. Faria CRS, et al, Fregonesi CEP, Corazza DAG, Andrade, SM, Manotvani NAD, Silva JR. Grau de incapacidade física de portadores de hanseníase: estudo de coorte retrospectivo. Arquivos de Ciências da Saúde. 2015;22(4):58-62.

22. Sousa ALG. Episódios reacionais em pacientes acometidos pela hanseníase em município endêmico [dissertação]. Piauí: Universidade Federal do Piauí; 2018.

23. Cunha ATR, Vilar RLA, Melo RHV, Silva AB, Rodrigues MP. Percepções de usuários sobre humanização na estratégia saúde da família: um estudo ancorado na teoria da Dádiva. Rev. Ciên. Plu. 2017;3(3):16-31. 